\title{
Zeeman Splitting and Inverted Polarization of Biexciton Emission in Monolayer $\mathbf{W S}_{2}$
}

\author{
Philipp Nagler, ${ }^{1, *}$ Mariana V. Ballottin, ${ }^{2}$ Anatolie A. Mitioglu, ${ }^{2}$ Mikhail V. Durnev, ${ }^{3}$ Takashi Taniguchi, ${ }^{4}$ Kenji Watanabe, \\ Alexey Chernikov, ${ }^{1}$ Christian Schüller, ${ }^{1}$ Mikhail M. Glazov, ${ }^{3}$ Peter C. M. Christianen, ${ }^{2}$ and Tobias Korn ${ }^{1, \dagger}$ \\ ${ }^{1}$ Department of Physics, University of Regensburg, D-93040 Regensburg, Germany \\ ${ }^{2}$ High Field Magnet Laboratory (HFML-EMFL), Radboud University, 6525 ED Nijmegen, The Netherlands \\ ${ }^{3}$ Ioffe Institute, 194021 St. Petersburg, Russia \\ ${ }^{4}$ National Institute for Materials Science, Tsukuba, Ibaraki 305-004, Japan
}

(Received 17 January 2018; revised manuscript received 23 April 2018; published 2 August 2018)

\begin{abstract}
Atomically thin semiconductors provide an ideal testbed to investigate the physics of Coulomb-bound many-body states. We shed light on the intricate structure of such complexes by studying the magneticfield-induced splitting of biexcitons in monolayer $\mathrm{WS}_{2}$ using polarization-resolved photoluminescence spectroscopy in out-of-plane magnetic fields up to $30 \mathrm{~T}$. The observed $g$ factor of the biexciton amounts to about -3.9 , closely matching the $g$ factor of the neutral exciton. The biexciton emission shows an inverted circular field-induced polarization upon linearly polarized excitation; i.e., it exhibits preferential emission from the high-energy peak in a magnetic field. This phenomenon is explained by taking into account the hybrid configuration of the biexciton constituents in momentum space and their respective energetic behavior in magnetic fields. Our findings reveal the critical role of dark excitons in the composition of this many-body state.
\end{abstract}

DOI: 10.1103/PhysRevLett.121.057402

Monolayer transition metal dichalcogenides (TMDCs) are a fascinating platform to study the physics of Coulombcorrelated quasiparticles in the two-dimensional limit. Because of reduced dimensionality and dielectric screening, excitons in these materials possess binding energies on the order of $0.5 \mathrm{eV}$, making them stable at room temperature and dominate the optical response [1-5]. Recently, experimental evidence for biexcitons, where two excitons bind to a four-particle state has been found in molybdenumand tungsten-based monolayer TMDCs [6-16]. These excitonic molecules are subject to intriguing many-body physics and could serve as a platform for quantum optics experiments due to their cascaded emission accompanied by entangled photon generation [17-19]. However, key questions with respect to the nature of the biexciton [20] and specifically the composition of this many-body state in momentum space remain open. In this respect, probing the behavior of excitonic complexes in strong magnetic fields has proven to be a powerful tool to gain a detailed understanding of these quasiparticles. Recently, this approach has revealed fundamental insights into neutral and charged excitons of atomically thin TMDCs [21-28]. Moreover, it was shown that the observed magnetic-fieldinduced polarization can be crucial for drawing conclusions on the composition of Coulomb-correlated states [29,30]. Therefore, we expect to obtain critical information such as the $g$ factor and the configuration of charge carriers comprising biexcitons in momentum space by studying these complex many-body states in high magnetic fields.
Here, we address these questions by probing biexcitons in monolayer $\mathrm{WS}_{2}$ in an external out-of-plane magnetic field up to $30 \mathrm{~T}$. The magnetic field lifts the valley degeneracy of the biexciton, allowing us to extract its spectroscopic $g$ factor of $-3.9 \pm 0.1$, in close agreement to the spectroscopic $g$ factor of the neutral exciton of $-3.8 \pm 0.1$ and thus providing further evidence for the concept of biexcitons in atomically thin TMDCs. Under linearly polarized excitation, we observe an inverted polarization of the biexciton emission in the magnetic field, implying that the state emitting at the higher energy is preferentially occupied, in contrast to the behavior of the neutral exciton. These observations, together with the theoretical analysis, allow us to draw conclusions on the valley configuration of the biexciton in $\mathrm{WS}_{2}$, inferring that it consists of a bright exciton in one valley and an intravalley dark exciton in the other valley.

The sample in this study [see Fig. 1(a)] was fabricated by an all-dry transfer technique [31] and consists of a monolayer of $\mathrm{WS}_{2}$ (bulk crystals from HQ Graphene) which is sandwiched between two thin sheets of hexagonal boron nitride (hBN). Steady-state photoluminescence (PL) measurements were performed using excitation by a continuous-wave laser with a photon energy of $2.21 \mathrm{eV}$. The laser was focused by an objective to a spot size of about $4 \mu \mathrm{m}$. The collected PL signal was analyzed by a spectrometer equipped with a liquid-nitrogen-cooled CCD. All experiments presented in the main manuscript have been conducted at a nominal sample temperature of $4.5 \mathrm{~K}$. 
(a)

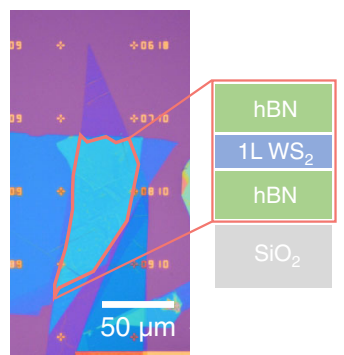

(c)
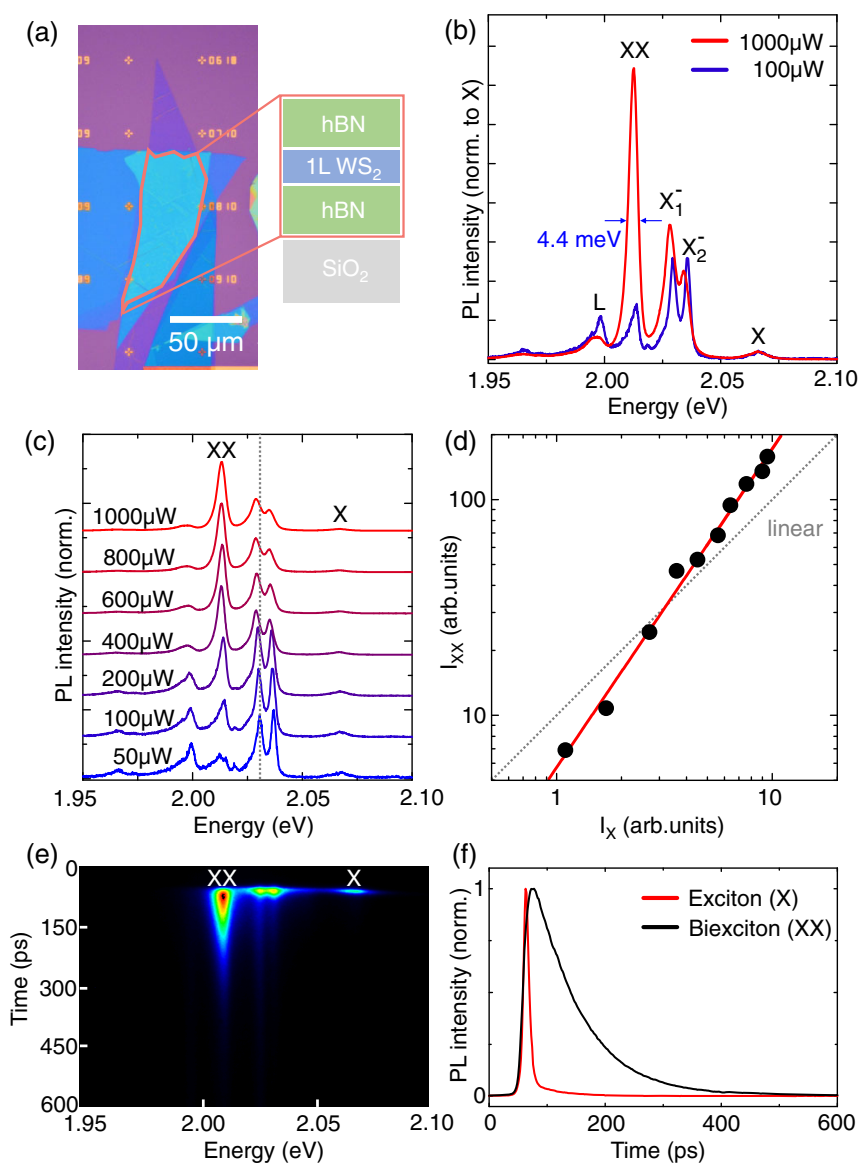

FIG. 1. (a) Micrograph of the $\mathrm{hBN} / \mathrm{WS}_{2} / \mathrm{hBN} / \mathrm{SiO}_{2}$ heterostructure. (b) PL spectra at $4 \mathrm{~K}$ under excitation powers of 100 (blue) and $1000 \mu \mathrm{W}$ (red). The spectra are normalized to the intensity of the neutral exciton $(X)$. (c) PL spectra at $4 \mathrm{~K}$ for different excitation powers. The dashed line marks the energetic position of the low-energy trion state $\left(X_{1}^{-}\right)$at $50 \mu \mathrm{W}$. The spectra are normalized to the peak with the highest intensity. (d) Double logarithmic representation of the intensity of the biexciton $(X X)$ as a function of the intensity of the neutral exciton. The red line is a power-law fit with $I_{X X} \sim\left(I_{X}\right)^{\alpha}$ with $\alpha=1.48$. The gray dashed line indicates a linear relation. (e) False-color plot of TRPL spectrum measured at $4 \mathrm{~K}$ and $40 \mu \mathrm{W}$. (f) Normalized TRPL traces of the $X$ and $X X$ features extracted from (e).

A PL spectrum of the structure at an excitation power of $100 \mu \mathrm{W}$ is shown in Fig. 1(b) (blue line). In agreement with recent reports, the encapsulation of the monolayer TMDC with hBN results in significantly reduced linewidths [32-37]. In the spectrum we can clearly resolve the neutral exciton $(X)$ at $2.067 \mathrm{eV}$ and the two trion species $X_{1}^{-}$ and $X_{2}^{-}$at an energy of 2.030 and $2.036 \mathrm{eV}$ [38-41]. Furthermore, at an energy of $1.998 \mathrm{eV}$ we observe emission which is typically attributed to the recombination of carriers localized at defects $(L)$. The red line in Fig. 1(b) depicts the spectrum with an excitation power of $1000 \mu \mathrm{W}$, normalized to the intensity of the neutral exciton $X$. The intensity of the two trion features scales like the exciton intensity and the emission from the defect state $L$ saturates. Most importantly, at this high excitation power, a novel feature emerges at $2.012 \mathrm{eV}$ exhibiting a clear superlinear behavior. We attribute this peak to the emission of biexcitons $(X X)$, in line with recent literature $[6-8,13,14]$ with a biexciton binding energy of about $53 \mathrm{meV}$ (see discussion in the Supplemental Material [42] for an alternative interpretation of this peak as a charged biexciton, which does not affect the main conclusions of our study). The superlinear increase of the biexciton peak is illustrated in Fig. 1(c) where spectra normalized to the intensity of the maximum peak for different excitation powers are depicted. Relating the intensity of the biexciton with the intensity of the neutral exciton with $I_{X X} \sim\left(I_{X}\right)^{\alpha}$ yields a factor $\alpha$ of 1.48 ; see Fig. 1(d). While $\alpha=2$ would be expected for full thermal equilibrium between neutral exciton and biexciton, values for $\alpha$ smaller than 2 have been regularly observed in TMDCs and were linked to a lack of full equilibrium between the two states [6].

To track the dynamics of neutral exciton and biexciton we employ a streak camera combined with a pulsed laser at an excitation energy of $2.21 \mathrm{eV}$. The time-resolved traces are shown in Figs. 1(e) and 1(f). While the decay of the neutral excitons is faster than our system resolution (10 ps), the biexciton decay can be readily quantified. A monoexponential fit yields a decay time of 83 ps for the biexciton. A possible contribution to a slower decay rate of the biexciton PL may be explained within the excitonic molecule model of Ref. [51], which predicts the reduction of the biexciton radiative decay rate as compared to the exciton rate roughly by a factor $\mu \sim(q a)^{2} \ll 1$, where $q$ is the wave vector of light inside a monolayer and $a$ is the characteristic interexcitonic separation within the biexciton; see Supplemental Material [42].

We now turn to measurements of the biexciton and exciton spectra in an out-of-plane magnetic field of up to 30 T. All measurements in magnetic field have been carried out with a linearly polarized laser, populating both valley configurations equally (see Supplemental Material [42] for a discussion on circularly polarized excitation conditions). The emission is analyzed in a circularly polarized basis, which allows us to resolve the resulting magnetic splitting and to quantify the degree of polarization. Figures 2(a) and 2(b) show spectra of the $X X$ and $X$ peak at $0,10,20$, and $30 \mathrm{~T}$ for both detection polarizations. The fieldinduced energetic splitting for both exciton and biexciton amounts to $\approx 6.7 \mathrm{meV}$ at $30 \mathrm{~T}$ for both features. The resulting energetic splitting of the $\sigma^{ \pm}$-polarized emission peaks with energies $E^{\sigma \pm}$ for exciton and biexciton is shown in Fig. 2(c). Using the definition

$$
\Delta E=E^{\sigma^{+}}-E^{\sigma^{-}}=g_{S} \mu_{B} B,
$$

where $\mu_{B} \approx 58 \mu \mathrm{eV} / \mathrm{T}$ is the Bohr magneton, $B$ is the magnetic field, and $g_{S}$ is the spectroscopic $g$ factor of the emitting state, we obtain $g_{S}^{X X}=-3.9 \pm 0.1$ for the 
(a)

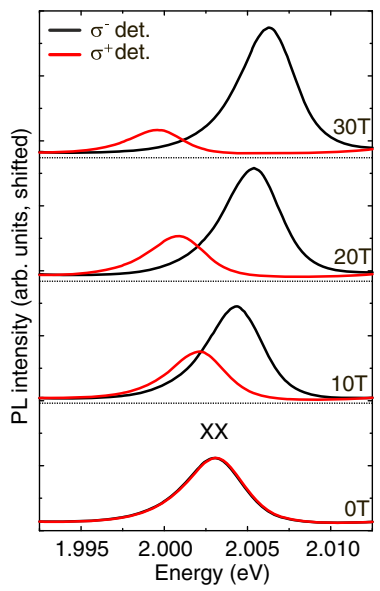

(c)

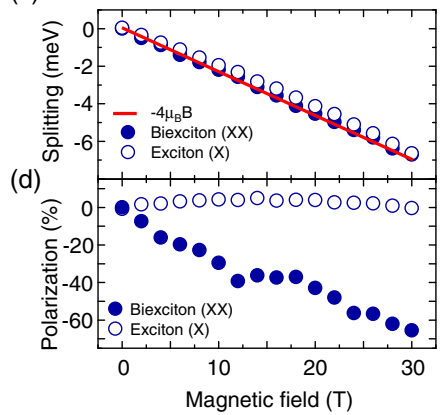

(b)

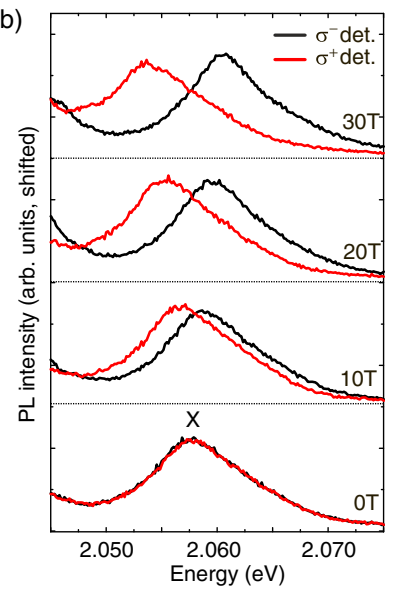

(e)

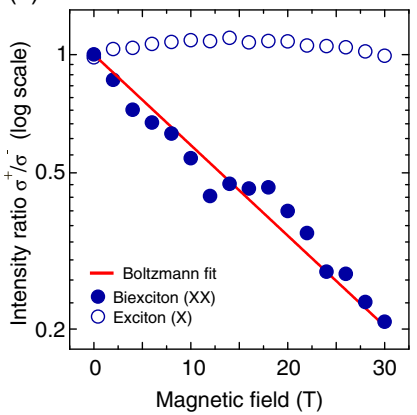

FIG. 2. (a) PL spectra of the $X X$ peak for $\sigma^{+}$and $\sigma^{-}$polarized detection after excitation with linearly polarized light for various out-of-plane magnetic fields up to $30 \mathrm{~T}$. (b) Same as in (a) but for the $X$ peak. (c) Peak splitting of the $X$ peak (open circles) and the $X X$ peak (filled circles) in dependence of magnetic field. The solid red line indicates a linear dependence with a $g$ factor of -4 . (d) Magnetic-field-induced polarization of the exciton and the biexciton. (e) Corresponding intensity ratio (log. scale) of the $\sigma^{+}$ and $\sigma^{-}$polarized light components in detection. The solid red line is a fit to a thermal Boltzmann distribution, Eq. (5), with $g_{T}^{X X}=4$ and $T=50 \mathrm{~K}$.

biexciton. The deduced spectroscopic $g$ factor of the neutral exciton amounts to $g_{S}^{X}=-3.8 \pm 0.1$, in very close agreement to previous measurements on $\mathrm{WS}_{2}[27,28,52]$ and almost identical to that of the biexciton.

However, the intensities of the circular emission components behave distinctively different for the $X$ and $X X$ emission at high magnetic fields. The corresponding fieldinduced circular polarization, defined as $P=\left(I_{\sigma^{+}}-I_{\sigma^{-}}\right) /$ $\left(I_{\sigma^{+}}+I_{\sigma^{-}}\right)$, where $I_{\sigma^{+/-}}$is obtained by a spectrally integrated Gaussian fit of the respective peak, is shown in Fig. 2(d). For the exciton, the emission is mostly unpolarized, but for the biexciton the intensity of the energetically higher polarization component $\left(\sigma^{-}\right.$for $\left.B>0\right)$ is strongly increased as compared with the lower-energy component and reaches values close to $-70 \%$ at $30 \mathrm{~T}$. A similar depiction is given in Fig. 2(e) where the ratio of the two intensities $I_{\sigma^{+}} / I_{\sigma^{-}}$is plotted vs the magnetic field, which will be discussed in more detail below.

The absence of circular polarization at the exciton resonance may be understood as a result of its very short, $\lesssim 10 \mathrm{ps}$, lifetime, during which excitons are likely to be far from a quasiequilibrium, and the off-resonant excitation conditions in our experiment. However, the observation of an inverted polarization of biexcitons, where the state emitting at high energy is more populated, is less obvious. Central to the understanding of this result is the concept that the distribution of excitonic complexes between energetically split levels in a magnetic field is determined by the change of the total energy of the state and not only of the component that is responsible for the observed emission feature. Similar effects were observed in magneto-PL studies on charged excitonic complexes in CdTe quantum wells $[29,30]$.

To take this general notion into account, we introduce an additional $g$ factor besides the spectroscopic $g$ factor $g_{S}$, which we call the total $g$ factor $g_{T}$. In contrast to $g_{S}$, which is only related to the energy of the emitting state, $g_{T}$ describes the Zeeman shifts of all constituents of the biexciton, i.e., the total energy of the composite exciton state. Hence, assuming quasiequilibrium, the ratio of the two intensities $I_{\sigma^{+}} / I_{\sigma^{-}}$that reflects the relative populations, which is therefore directly linked to the total energy of the excitonic state can also be expressed by means of a Boltzmann distribution as $I_{\sigma^{+}} / I_{\sigma^{-}}=\exp \left(-g_{T} \mu_{B} B / k_{B} T\right)$, where $T$ is the effective temperature of the biexciton gas and $k_{B}$ is the Boltzmann constant. An inverted polarization behavior, where $I_{\sigma^{-}}<I_{\sigma^{+}}$holds, can thus be expected if $g_{T}>0$. On the other hand, the spectroscopic $g$ factor $g_{S}$ only refers to the energies of the emitting state. Hence, for an exciton we always have $g_{S}=g_{T}$, since the emitting state is identical to the total excitonic complex. However, for more complex states such as the biexciton, the total $g$ factor $g_{T}$ can differ from $g_{S}$ as it contains also the energies of the nonemitting components. Our analysis below shows that in the case of biexcitons in monolayer $\mathrm{WS}_{2}$, the total energies of the states are not identical to the emission energies due to the presence of the second, dark exciton in this four-particle complex giving rise to the observed inverted polarization.

To elucidate the expected polarization behavior of biexcitons in a magnetic field, let us consider different configurations of the biexciton in $\mathrm{WS}_{2}$ without magnetic field. Here, we adopt the conventional picture of the biexciton state in $\mathrm{WS}_{2}$ as being charge neutral and originating from two excitons in the $\boldsymbol{K}$ valleys. In the ground state $X X_{1}$, as shown in the upper part of Fig. 3(a), the two electrons occupy the lowest-lying conduction bands of the $\boldsymbol{K}_{+}$and $\boldsymbol{K}_{-}$valleys, respectively, and the holes the highest-lying valence bands. While this arrangement is energetically the most favorable, it is optically dark since intravalley electron-hole transitions are spin forbidden in this case; see Supplemental Material [42] for further discussion on $X X_{1}$ and the highly excited state $X X_{4}$. Thus, we do not detect this configuration in our optical 
(a)

$\mathbf{X X}_{1}$ (Ground state)
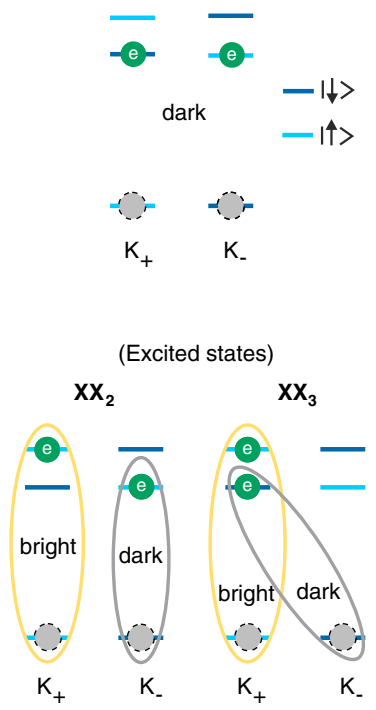

(b)

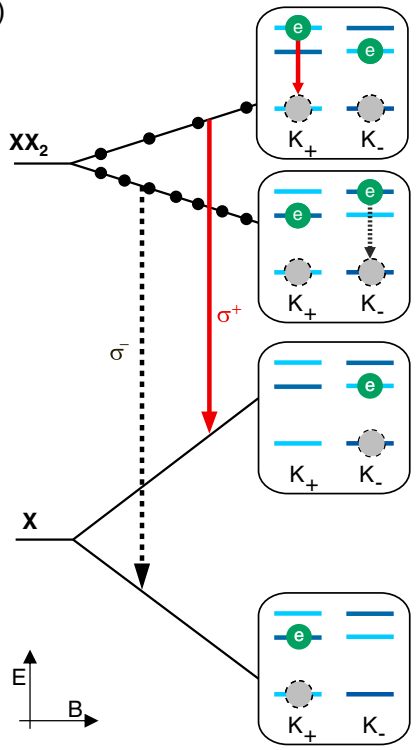

FIG. 3. (a) Three configurations of biexcitons in monolayer $\mathrm{WS}_{2}$. Both excited states, $X X_{2}$ and $X X_{3}$, have one transition which is both spin- and momentum-allowed. The remaining dark state is either spin-forbidden $\left(X X_{2}\right)$ or indirect in momentum space $\left(X X_{3}\right)$. Green circles denote electrons in the conduction band and grey circles denote unoccupied states in the valence band. (b) Schematics of biexciton $X X_{2}$ recombination showing that the lower-energy state of $X X_{2}$ emits at a higher photon energy in emission. The number of dots sketches relative populations of the Zeeman-split $X X_{2}$ levels. Solid and dashed arrows denote recombination paths in $\sigma^{+}$and $\sigma^{-}$polarization, respectively.

experiments. The other two configurations in Fig. 3(a), $X X_{2}$ and $X X_{3}$, consist of one bright electron-hole pair, which can recombine with emission of a photon, and one dark electron-hole pair, which is optically inactive.

The Zeeman splitting of biexciton states can be analyzed by considering the corresponding shifts of the conduction and valence bands. The Zeeman Hamiltonian for an electron in the conduction $c$ or valence band $v$ reads

$$
\mathcal{H}_{c, v}=\frac{1}{2} \mu_{B} B\left(g_{c, v}^{\mathrm{orb}} \tau_{z}+g_{c, v}^{\mathrm{sp}} \sigma_{z}\right)
$$

where $g^{\text {orb }}$ is the $g$ factor describing orbital contributions to the Zeeman splitting ( $\tau_{z}= \pm 1$ is the valley index), and $g^{\text {sp }}$ describes the spin contribution $\left(\sigma_{z}= \pm 1\right.$ is the spin index), which is largely unaffected by the crystal environment and thus set to the vacuum value of 2 for both conduction and valence bands [53]. Since the radiative recombination pathway of the biexciton states $X X_{2}$ and $X X_{3}$ is essentially identical to that of the optically bright exciton, the spectroscopic $g$ factor of these complexes, introduced in Eq. (1), is the same as of the bright exciton, and equals to

$$
g_{S}^{X X}=g_{S}^{X}=g_{c}^{\mathrm{orb}}+g_{c}^{\mathrm{sp}}-g_{v}^{\mathrm{orb}}-g_{v}^{\mathrm{sp}} .
$$

Using recently calculated values for $\mathrm{WS}_{2}$ [53] with $g_{v}^{\text {orb }}=$ 3.96 and $g_{c}^{\text {orb }}=0.11$ and setting $g_{c}^{\mathrm{sp}}=g_{v}^{\mathrm{sp}}=2$ we obtain a theoretical value of $g_{\mathrm{th}}^{X X}=-3.85$. This is in good agreement with the experimental observation of $g_{S}^{X}=-3.8 \pm 0.1$ and $g_{S}^{X X}=-3.9 \pm 0.1$. Note that these estimates are also consistent with the approach of Refs. [21-23].

The distribution of biexcitons over the Zeeman-split states is determined, however, by the total energy of the biexciton which accounts for both the energy of the bright and dark exciton. Correspondingly, the total Zeeman splitting of a biexciton state, proportional to the total $g$ factor $g_{T}^{X X}$, differs from the spectroscopic $g$ factor, $g_{S}^{X X}$. Both $X X_{2}$ and $X X_{3}$ states consist of a pair of holes in a singlet state which is unaffected by the magnetic field and a pair of electrons with the same spins located in different valleys $\left(X X_{2}\right)$ or in the same valley but with opposite spins $\left(X X_{3}\right)$ yielding

$$
g_{T}^{X X_{2}}=2 g_{c}^{\mathrm{sp}} \approx+4, \quad g_{T}^{X X_{3}}=2 g_{c}^{\mathrm{orb}} \approx+0.2,
$$

where the estimates have been obtained taking $g_{c}^{\text {orb }}$ from Ref. [53]. In this case, where $g_{T}^{X X}$ is positive, the $\sigma^{-}$-active biexciton state is energetically lower than the $\sigma^{+}$-active one, and hence is more populated, leading to an inverted polarization. The expected evolution of energy levels and the corresponding population for this situation are depicted in Fig. 3(b). One can also arrive at Eq. (4) by summing up the Zeeman splittings of bright and dark excitons, see Supplemental Material [42]. We also note here that the observed population imbalance of the biexciton states results from intervalley scattering processes to the energetically lowest level [54].

According to the estimates in Eq. (4) both $X X_{2}$ and $X X_{3}$ biexcitons lead to inverted polarization. In a simplified picture we can assume that biexcitons are distributed between the two states in a quasiequilibrium during emission. Further insight is given by the fit of the experimental data in Fig. 2(e) with the following equation:

$$
\frac{I_{\sigma^{+}}}{I_{\sigma^{-}}}=\exp \left(-\frac{g_{T}^{X X} \mu_{B} B}{k_{B} T}\right) .
$$

From this fit we can extract only the ratio of the total $g$ factor $g_{T}$ and the effective temperature of the biexciton gas $T$. Using theoretical estimations for $g_{T}$ given by Eq. (4) we obtain an effective temperature $T \approx 50 \mathrm{~K}$ for the $X X_{2}$ complex and $T \approx 2.5 \mathrm{~K}$ for the $X X_{3}$ complex. The fact that in the latter case the value of $T$ is lower than the nominal sample temperature in our setup of $4.5 \mathrm{~K}$ strongly indicates that the inverted polarization is provided by the $X X_{2}$ biexciton. The expected polarization behavior in the alternative scenario of a charged biexciton in a magnetic field is discussed in the Supplemental Material [42].

The deviation of the effective temperature $T$ from the nominal sample temperature can stem either from the 
biexciton gas being out of full thermal equilibrium with the lattice due to slow cooling by acoustic phonons or from the heating of the crystal itself due to laser excitation with high power $(1000 \mu \mathrm{W})$. The latter effect can be estimated by determining the power-induced energetic redshift of the trion which amounts to $2.1 \mathrm{meV}$ at $1000 \mu \mathrm{W}$, see dashed line in Fig. 1(c). Comparing this shift to the typical temperature dependence of the peak energies of the sample with increasing temperature we estimate a lattice temperature of $35-55 \mathrm{~K}$, in good qualitative agreement with the temperature from the Boltzmann fit (see Supplemental Material [42] for details).

In conclusion, we have measured the polarizationresolved photoluminescence of biexcitons and excitons in monolayer $\mathrm{WS}_{2}$ in a perpendicular magnetic field up to $30 \mathrm{~T}$. We determine the spectroscopic $g$ factor of the biexciton to be $g_{S}^{X X}=-3.9 \pm 0.1$, closely matching the spectroscopic $g$ factor of the exciton $g_{S}^{X}=-3.8 \pm 0.1$. Interestingly, we have observed that the sign of magneto-induced circular polarization does not match the sign of the Zeeman splitting of biexciton emission. This observation is explained by taking into account the evolution of the total energy of the biexciton in a magnetic field. On the basis of the experiment and the developed model we are able to identify the optically dominant excited biexciton state of monolayer $\mathrm{WS}_{2}$. Our results form a basis for future experiments on these many-body states and highlight the importance of the dark states involved in the formation of biexcitons.

Financial support by the DFG via GRK 1570, KO 3612/11, KO 3612/3-1, SFB 689, SFB 1277 (B05) and CH 1672/11 and support of HFML-RU/FOM, member of the European Magnetic Field Laboratory (EMFL) is gratefully acknowledged. M. V. D. acknowledges financial support from RFBR Project No. 16-32-60175 and the Russian Federation (RF) President Grant No. MK-7389.2016.2. M. M. G. was partially supported by RF President Grant No. MD-1555.2017.2. Growth of hexagonal boron nitride crystals was supported by the Elemental Strategy Initiative conducted by the MEXT, Japan and JSPSKAKENHI Grant No. JP15K21722.

Note added in proof.-Recently, we became aware of several works studying biexciton and charged biexciton states in monolayers of the related material tungsten diselenide, in which a similar inverted polarization of the biexciton emission in an out-of-plane magnetic field was observed [55-57].

*philipp.nagler@ur.de tobias.korn@ur.de

[1] K. He, N. Kumar, L. Zhao, Z. Wang, K. F. Mak, H. Zhao, and J. Shan, Phys. Rev. Lett. 113, 026803 (2014).

[2] M. M. Ugeda, A. J. Bradley, S.-F. Shi, F. H. da Jornada, Y. Zhang, D. Y. Qiu, W. Ruan, S.-K. Mo, Z. Hussain, Z.-X.
Shen, F. Wang, S. G. Louie, and M. F. Crommie, Nat. Mater. 13, 1091 (2014).

[3] A. Chernikov, T. C. Berkelbach, H. M. Hill, A. Rigosi, Y. Li, O. B. Aslan, D. R. Reichman, M. S. Hybertsen, and T. F. Heinz, Phys. Rev. Lett. 113, 076802 (2014).

[4] Z. Ye, T. Cao, K. O’Brien, H. Zhu, X. Yin, Y. Wang, S. G. Louie, and X. Zhang, Nature (London) 513, 214 (2014).

[5] M. Van der Donck, M. Zarenia, and F. M. Peeters, Phys. Rev. B 96, 035131 (2017).

[6] Y. You, X.-X. Zhang, T. C. Berkelbach, M. S. Hybertsen, D. R. Reichman, and T. F. Heinz, Nat. Phys. 11, 477 (2015).

[7] J. Shang, X. Shen, C. Cong, N. Peimyoo, B. Cao, M. Eginligil, and T. Yu, ACS Nano 9, 647 (2015).

[8] G. Plechinger, P. Nagler, J. Kraus, N. Paradiso, C. Strunk, C. Schüller, and T. Korn, Phys. Status Solidi-Rapid Res. Lett. 9, 457 (2015).

[9] E. J. Sie, A. J. Frenzel, Y.-H. Lee, J. Kong, and N. Gedik, Phys. Rev. B 92, 125417 (2015).

[10] E. J. Sie, C. H. Lui, Y.-H. Lee, J. Kong, and N. Gedik, Nano Lett. 16, 7421 (2016).

[11] H. S. Lee, M. S. Kim, H. Kim, and Y. H. Lee, Phys. Rev. B 93, 140409 (2016).

[12] M. S. Kim, S. J. Yun, Y. Lee, C. Seo, G. H. Han, K. K. Kim, Y. H. Lee, and J. Kim, ACS Nano 10, 2399 (2016).

[13] M. Okada, Y. Miyauchi, K. Matsuda, T. Taniguchi, K. Watanabe, H. Shinohara, and R. Kitaura, Sci. Rep. 7, 322 (2017).

[14] I. Paradisanos, S. Germanis, N. T. Pelekanos, C. Fotakis, E. Kymakis, G. Kioseoglou, and E. Stratakis, Appl. Phys. Lett. 110, 193102 (2017).

[15] K. Hao, J. F. Specht, P. Nagler, L. Xu, K. Tran, A. Singh, C. K. Dass, C. Schüller, T. Korn, M. Richter, A. Knorr, X. Li, and G. Moody, Nat. Commun. 8, 15552 (2017).

[16] J. Pei, J. Yang, X. Wang, F. Wang, S. Mokkapati, T. Lü, J.-C. Zheng, Q. Qin, D. Neshev, H. H. Tan, C. Jagadish, and Y. Lu, ACS Nano 11, 7468 (2017).

[17] O. Benson, C. Santori, M. Pelton, and Y. Yamamoto, Phys. Rev. Lett. 84, 2513 (2000).

[18] R. J. Young, R. M. Stevenson, P. Atkinson, K. Cooper, D. A. Ritchie, and A. J. Shields, New J. Phys. 8, 29 (2006).

[19] Y.-M. He, O. Iff, N. Lundt, V. Baumann, M. Davanco, K. Srinivasan, S. Höfling, and C. Schneider, Nat. Commun. 7, 13409 (2016).

[20] D. Van Tuan, B. Scharf, I. Žutić, and H. Dery, Phys. Rev. X 7, 041040 (2017).

[21] G. Aivazian, Z. Gong, A. M. Jones, R.-L. Chu, J. Yan, D. G. Mandrus, C. Zhang, D. Cobden, W. Yao, and X. Xu, Nat. Phys. 11, 148 (2015).

[22] Y. Li, J. Ludwig, T. Low, A. Chernikov, X. Cui, G. Arefe, Y. D. Kim, A. M. van der Zande, A. Rigosi, H. M. Hill, S. H. Kim, J. Hone, Z. Li, D. Smirnov, and T. F. Heinz, Phys. Rev. Lett. 113, 266804 (2014).

[23] A. Srivastava, M. Sidler, A. V. Allain, D. S. Lembke, A. Kis, and A. Imamoğlu, Nat. Phys. 11, 141 (2015).

[24] D. MacNeill, C. Heikes, K. F. Mak, Z. Anderson, A. Kormányos, V. Zólyomi, J. Park, and D. C. Ralph, Phys. Rev. Lett. 114, 037401 (2015).

[25] G. Wang, L. Bouet, M. M. Glazov, T. Amand, E. L. Ivchenko, E. Palleau, X. Marie, and B. Urbaszek, 2D Mater. 2, 034002 (2015). 
[26] A. A. Mitioglu, P. Plochocka, Á. Granados del Aguila, P. C. M. Christianen, G. Deligeorgis, S. Anghel, L. Kulyuk, and D. K. Maude, Nano Lett. 15, 4387 (2015).

[27] A. V. Stier, K. M. McCreary, B. T. Jonker, J. Kono, and S. A. Crooker, Nat. Commun. 7, 10643 (2016).

[28] G. Plechinger, P. Nagler, A. Arora, A. Granados del Águila, M. V. Ballottin, T. Frank, P. Steinleitner, M. Gmitra, J. Fabian, P. C. M. Christianen, R. Bratschitsch, C. Schüller, and T. Korn, Nano Lett. 16, 7899 (2016).

[29] G. V. Astakhov, D. R. Yakovlev, V. V. Rudenkov, P. C. M. Christianen, T. Barrick, S. A. Crooker, A. B. Dzyubenko, W. Ossau, J. C. Maan, G. Karczewski, and T. Wojtowicz, Phys. Rev. B 71, 201312 (2005).

[30] G. Bartsch, M. Gerbracht, D. R. Yakovlev, J. H. Blokland, P. C. M. Christianen, E. A. Zhukov, A. B. Dzyubenko, G. Karczewski, T. Wojtowicz, J. Kossut, J. C. Maan, and M. Bayer, Phys. Rev. B 83, 235317 (2011).

[31] A. Castellanos-Gomez, M. Buscema, R. Molenaar, V. Singh, L. Janssen, H. S. J. van der Zant, and G. A. Steele, 2D Mater. 1, 011002 (2014).

[32] Z. Wang, J. Shan, and K. F. Mak, Nat. Nanotechnol. 12, 144 (2016).

[33] Z. Wang, L. Zhao, K. F. Mak, and J. Shan, Nano Lett. 17, 740 (2017).

[34] F. Cadiz, E. Courtade, C. Robert, G. Wang, Y. Shen, H. Cai, T. Taniguchi, K. Watanabe, H. Carrere, D. Lagarde, M. Manca, T. Amand, P. Renucci, S. Tongay, X. Marie, and B. Urbaszek, Phys. Rev. X 7, 021026 (2017).

[35] M. Manca, M. M. Glazov, C. Robert, F. Cadiz, T. Taniguchi, K. Watanabe, E. Courtade, T. Amand, P. Renucci, X. Marie, G. Wang, and B. Urbaszek, Nat. Commun. 8, 14927 (2017).

[36] O. A. Ajayi, J. V. Ardelean, G. D. Shepard, J. Wang, A. Antony, T. Taniguchi, K. Watanabe, T. F. Heinz, S. Strauf, X.-Y. Zhu, and J. C. Hone, 2D Mater. 4, 031011 (2017).

[37] J. Wierzbowski, J. Klein, F. Sigger, C. Straubinger, M. Kremser, T. Taniguchi, K. Watanabe, U. Wurstbauer, A. W. Holleitner, M. Kaniber, K. Müller, and J. J. Finley, Sci. Rep. 7, 12383 (2017).

[38] H. Yu, G.-B. Liu, P. Gong, X. Xu, and W. Yao, Nat. Commun. 5, 3876 (2014).
[39] A. M. Jones, H. Yu, J. R. Schaibley, J. Yan, D. G. Mandrus, T. Taniguchi, K. Watanabe, H. Dery, W. Yao, and X. Xu, Nat. Phys. 12, 323 (2016).

[40] G. Plechinger, P. Nagler, A. Arora, R. Schmidt, A. Chernikov, A. G. del Águila, P. C. M. Christianen, R. Bratschitsch, C. Schüller, and T. Korn, Nat. Commun. 7, 12715 (2016).

[41] E. Courtade, M. Semina, M. Manca, M. M. Glazov, C. Robert, F. Cadiz, G. Wang, T. Taniguchi, K. Watanabe, M. Pierre, W. Escoffier, E. L. Ivchenko, P. Renucci, X. Marie, T. Amand, and B. Urbaszek, Phys. Rev. B 96, 085302 (2017).

[42] See Supplemental Material at http://link.aps.org/ supplemental/10.1103/PhysRevLett.121.057402, which includes Refs. [43-50], for additional experimental details and theoretical analysis.

[43] P. Varshni, Physica (Amsterdam) 34, 149 (1967).

[44] L. Viña, S. Logothetidis, and M. Cardona, Phys. Rev. B 30, 1979 (1984).

[45] R. Pässler, Phys. Status Solidi 200, 155 (1997).

[46] I. Kylänpää and H.-P. Komsa, Phys. Rev. B 92, 205418 (2015).

[47] D. K. Zhang, D. W. Kidd, and K. Varga, Nano Lett. 15, 7002 (2015).

[48] M. Szyniszewski, E. Mostaani, N. D. Drummond, and V. I. Fal'ko, Phys. Rev. B 95, 081301 (2017).

[49] M. Danovich, V. Zólyomi, and V. I. Fal'ko, Sci. Rep. 7, 45998 (2017).

[50] C. Robert, D. Lagarde, F. Cadiz, G. Wang, B. Lassagne, T. Amand, A. Balocchi, P. Renucci, S. Tongay, B. Urbaszek, and X. Marie, Phys. Rev. B 93, 205423 (2016).

[51] D. S. Citrin, Phys. Rev. B 50, 17655 (1994).

[52] J. Kuhnert, A. Rahimi-Iman, and W. Heimbrodt, J. Phys. Condens. Matter 29, 08LT02 (2017).

[53] D. V. Rybkovskiy, I. C. Gerber, and M. V. Durnev, Phys. Rev. B 95, 155406 (2017).

[54] M. M. Glazov, T. Amand, X. Marie, D. Lagarde, L. Bouet, and B. Urbaszek, Phys. Rev. B 89, 201302 (2014).

[55] S.-Y. Chen, T. Goldstein, T. Taniguchi, K. Watanabe, and J. Yan, arXiv:1802.10247.

[56] M. Barbone, et al., arXiv:1805.04950.

[57] Z. Li, et al., arXiv:1806.03775. 